\section{Regulating gene expression in differentiation}

from Carol K. Klukas

Consinering; the number of different cell types which exist in any mature eukaryotic organism, and the number of individual differentiation events which must have occurred in precisely ordered sequence in the course of its development one is left in no doubt that the system which so precisely controls genome expression in eukaryotes must be incredibly complex.

Research into many different aspects of the control system is currently in progress in many laboratories, and a recent paper from Strom and Dorfman (Proc. natn. Acad. Sci. U.S.A. 73, $3428 ; 1976)$ offers new evidence in support of the theory first put forward by Britten and Davidson, that the intermediate repetitive sequences in the genome may have a crucial role in regulating gene expression.

An early theory of the control of eukaryotic gene expression which proposed that appropriate structural genes are singled out and amplified during differentiation has generally proved to be untrue. Gene titration experiments have indicated only one or at most a few copies of any one structural gene, even in cell types where the protein that the gene specifies makes up a large proportion of the total protein synthesised. Research has therefore concentrated on the investigation of possible transcriptional controls, such as the interaction of non-histone proteins and hormones with chromatin, and the sequence organisation of the DNA itself. In 1969 Britten and Davidson (Science 165, 349; 1969) first suggested how, theoretically, intermediate repetitive sequences might control gene expression, and more recently (Davidson et al. Cell 4, 217; 1975) they have found that $80-100 \%$ of the mRNA molecules present in sea urchin embryos are transcribed from single copy DNA sequences which are adjacent to interspersed repetitive sequences in the genome. This finding does indeed suggest the regulatory importance of these sequences.

In a recent issue of Proc. natn. Acad. Sci. U.S.A. $(\mathbf{7 3}, 3428-3432 ; 1976)$ Strom and Dorfman offer new data which more directly supports this theory and which suggests a specific mechanism by which such intermediate repetitive sequences in the DNA may control differentiation. They use the chick limb bud system in which chick stage 24 limb bud mesenchymal cells are dissociated and cultured in vitro at high density. Once the cell density becomes greater than confluency these cells differentiate into cartilage unless the thymidine analogue 5-bromo-2'-deoxyuridine (BUdR) is present at a concentration of $32 \mathrm{mM}$ during the initial $48 \mathrm{~h}$ of culture in which case differentiation is irreversibly inhibited. In this study DNA was prepared from early cultures of these limb bud cells grown in ${ }^{3} \mathrm{H}-\mathrm{BUdR}$. The reassociation rate of this DNA was then measured after shearing and in the presence of a 100-fold excess of DNA prepared from cells at various stages of cartilage differentiation or from liver tissue or from whole chick embryos. These experiments show that sternal cartilage DNA and 8-day cultured limb bud cartilage DNA drive the reassociation reaction about twice as fast as does liver DNA or undifferentiated limb bud DNA or DNA from 8-day limb bud cultures blocked from differentiation by BUdR treatment. Furthermore, by analysing the reassociation kinetics of these reactions Strom and Dorfman determine that the total percentage of the tritium which reannealed in the moderately repetitive fraction was lower in liver DNA $(20 \%)$ than in sternal cartilage DNA $(30 \%)$, 8-day limb bud culture DNA $(30 \%)$, or in total embryonic DNA $(33 \%)$. These data suggest that in cells differentiated for cartilage production a certain set of intermediately repeated sequences is amplified, whereas in undifferentiated cells or in cells differentiated for other functions, this particular complement of intermediate repetitive sequences is not amplified.

Does this mean that the determinative event in the differentiation of cartilage cells might be the amplification of cartilage-specific sequences initially present in multiple copies? Several facts about this system suggest this to be true. First it must be noted that during the first $48 \mathrm{~h}$ of culture during which the differentiation process can be blocked by the presence of BUdR, very little cell division occurs. After $48 \mathrm{~h}$ the differentiation process is no longer sensitive to BUdR. Since it is known that the half life of ${ }^{3} \mathrm{H}-\mathrm{BUdR}$ in substituted DNA is 11 times shorter than ${ }^{3} \mathrm{H}-\mathrm{TdR}$, it stands to reason that the mechanism of BUdR inhibition of differentiation is that although the amplification of cartilage-specific intermediate repetitive DNA sequences does occur in these cultures during the first $48 \mathrm{~h}$, the amplification is accompanied bv and followed by rapid degradation of the sequences (because they contain BUdR) so that the cell does not receive the proper differentiation signal.

Similar BUdR sensitivity is exhibited by differentiation processes in various other chick cell types and in other tissues of diverse eukaryotic organisms.
Could it be that this proposed amplification event is a generalised feature of the cell differentiation process and that it is somehow involved in the regulation of genome expression? A positive answer to this question is certainly possible, but regardless of the outcome, further reports of research on this phenomenon are sure to be forthcoming soon and certainly promise to be exciting.

\section{Plasmids and pathogenicity}

\section{from David Sherratt}

ESCHERICHIA COLI has often been considered a harmless inhabitant both of the large intestine of man and animals and of molecular biology laboratories. However in the past ten years it has become increasingly clear that some strains of Escherichia coli can be pathogenic, Enteropathogenic strains in the gut cause diarrhoeal diseases and invasive strains cause bacteraemia and urinary infections. Diarrhoeal disease resulting from enteropathogenic $E$. coli may well be one of the leading causes of death of young children in developing countries (see Sack, $A$. Rev. Microbiol. 39, 333; 1975), and enteropathogenic $E$. coli cause considerable losses in intensively reared livestock.

Enteropathogenic $E$. coli strains produce either or both of two types of protein exotoxin, one heat stable (ST) the other heat labile (LT). The latter resembles cholera toxin and produces a similar but comparatively short-lived massive salt and water secretion from the mucosal cells of the small intestine -hence the diarrhoea. The similar effects of ST toxin are even more short-lived and the biochemical basis for its action at the cellular level is unknown. H. Williams Smith (J. gen. Microbiol. 52, 319; 1968) was the first to show that toxin production in $E$. coli is transmissible by conjugation, and this led to the finding that the toxins are plasmid specified, often by the same plasmid.

Little is known about the genetic determinants of toxins in other microorganisms, partly because little is known of their molecular biology. The genes for cholera toxin seem to be chromosomally located and the structural genes for diphtheria toxin are known to be located on a lysogenic bacteriophage. In addition, the genes responsible for causing crown gall tumour in plants seem to reside on a plasmid present in the causal microorganism Agrobacterium tumefaciens. 